\title{
High spatial resolution Galactic 3D extinction mapping with IPHAS
}

\author{
S. E. Sale ${ }^{1}$, J. E. Drew ${ }^{2}$ and the IPHAS Collaboration \\ ${ }^{1}$ Astrophysics Group, Imperial College London, Blackett Laboratory, Prince Consort Road, \\ London SW7 2AZ, U.K. \\ email: s.sale06@imperial.ac.uk \\ ${ }^{2}$ Centre for Astrophysics Research, STRI, University of Hertfordshire, College Lane Campus, \\ Hatfield, AL10 9AB, U.K.
}

\begin{abstract}
An algorithm, MEAD, is presented, which can map extinction in three dimensions, with fine distance and angular resolutions. MEAD is then employed when studying the structure of the outer Galaxy. We show that the Galaxy's radial density profile takes the form of a broken exponential, with density dropping off more steeply beyond a Galacto-centric radius of $\sim 13 \mathrm{kpc}$.
\end{abstract}

Keywords. surveys, Galaxy: disk, Galaxy: structure, Galaxy: stellar content, ISM: dust, extinction, ISM: structure

MEAD, can determine intrinsic $\left(r^{\prime}-i^{\prime}\right)$ colour, extinction, and distance for A0-K4 stars extracted from the IPHAS (www . iphas.org) $r^{\prime} / i^{\prime} / \mathrm{H} \alpha$ photometric database. These data can be binned up to map extinction in three dimensions across the northern Galactic Plane with fine angular $(\sim 10 \mathrm{arcmin})$ and distance $(\sim 0.1 \mathrm{kpc})$ resolution to distances of up to $10 \mathrm{kpc}$.

Subsequently study the stellar density profile of the outer Galactic disc in the anticentre direction. We select early A stars and compare observations with simulated photometry. By selecting A stars, we are appraising the properties of a population only $\sim 100$ Myrs old. We find the stellar density is well fit to an exponential with length scale of $\left(3020 \pm 120_{\text {statistical }} \pm 50_{R_{\odot}}\right)$ pc, out to a galacto-centric radius of $R_{T}=(13.0 \pm$ $\left.0.5_{\text {statistical }} \pm 0.2_{R_{\odot}}\right) \mathrm{kpc}$. At larger radii the rate of decline appears to increase with the scale length dropping to $(1200 \pm 300)$ pc, see Fig.1. This result amounts to a refinement of the conclusions reached in previous studies that the stellar density profile is abruptly truncated.

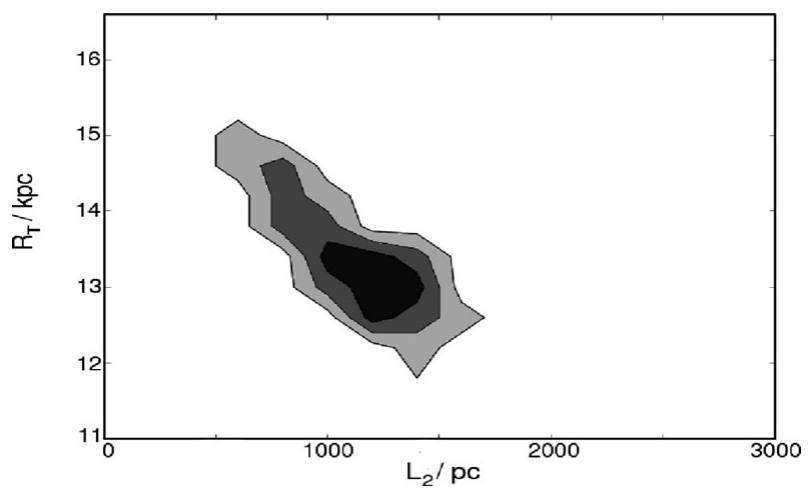

Figure 1. A contour plot showing the derived confidence limits on outer scale length and truncation radius. Contours are at $68 \%, 95 \%$ and $99 \%$. 